\title{
Stereoscopic depth perception during binocular rivalry
}

\section{Timothy J. Andrews* and David Holmes}

Department of Psychology and York Neuroimaging Centre, University of York, York, UK

\section{Edited by:}

Georgios A. Keliris, Max-Planck Institute for Biological Cybernetics, Germany

\section{Reviewed by:}

Shozo Tobimatsu, Kyushu University, Japan

Gijs Joost Brouwer, New York University, USA

*Correspondence:

Timothy J. Andrews, Department of Psychology and York Neuroimaging Centre, University of York, Heslington, York, Y010 5DD, UK.

e-mail: t.andrews@psych.york.ac.uk
When we view nearby objects, we generate appreciably different retinal images in each eye. Despite this, the visual system can combine these different images to generate a unified view that is distinct from the perception generated from either eye alone (stereopsis). However, there are occasions when the images in the two eyes are too disparate to fuse. Instead, they alternate in perceptual dominance, with the image from one eye being completely excluded from awareness (binocular rivalry). It has been thought that binocular rivalry is the default outcome when binocular fusion is not possible. However, other studies have reported that stereopsis and binocular rivalry can coexist. The aim of this study was to address whether a monocular stimulus that is reported to be suppressed from awareness can continue to contribute to the perception of stereoscopic depth. Our results showed that stereoscopic depth perception was still evident when incompatible monocular images differing in spatial frequency, orientation, spatial phase, or direction of motion engage in binocular rivalry. These results demonstrate a range of conditions in which binocular rivalry and stereopsis can coexist.

Keywords: stereopsis, binocular rivalry

\section{INTRODUCTION}

Theories of binocular integration are usually based around the phenomenon of stereopsis, which necessitates that the two monocular images are combined to form a single cyclopean image (Wheatstone, 1838; Julesz, 1971). The success of this conception is best exemplified by our current understanding of stereopsis, which depends on the convergence of monocular information onto disparity-sensitive binocular neurons that generate (or at least initiate) a sensation of depth (Cumming, 1997; Parker, 2007). However, the idea that binocular vision always involves integration of the two eye views is not easily reconciled with the experience of binocular rivalry (Alais and Blake, 2005). For example, if vertical stripes are presented to one eye and horizontal stripes are presented to a corresponding location in the other eye, the same region of visual space is perceived as being occupied by vertical stripes or horizontal stripes, but not by both. If the two monocular streams were simply united, one would presumably see a grid.

So, how can the visual system deal with monocular signals in such different ways? One possible explanation is that binocular rivalry is the default outcome when binocular correspondence cannot be solved (Blake, 1989; Lehky and Blake, 1991). In this model, rivalry results from reciprocal inhibition between monocular signals prior to binocular convergence. In the presence of well-matched monocular signals, the reciprocal inhibition responsible for rivalry is reduced by binocular neurons that generate the sensation of stereoscopic depth. However, if the modulating effect of the binocular circuitry is weakened by poorly matched stimuli, then the two monocular signals can engage in rivalry. Evidence in support of this model, comes from studies that show stereoscopic depth is disrupted during binocular rivalry (Blake et al., 1991; Harrad et al., 1994; Cogan et al., 1995).
The idea that binocular suppression only occurs when fusion is not possible is challenged by other studies that report that rivalry and stereopsis can coexist in the same location of the visual field (see Wolfe, 1986, for review). For example, it has been reported that stereoscopic depth is still evident when stereo targets are presented on a background undergoing rivalry (Treisman, 1962; Ogle and Wakefield, 1967; Harrad et al., 1994). Other studies have shown that random dot stereograms can still elicit a perception of depth in the presence of rivalrous noise (Julesz and Miller, 1975; Mayhew and Frisby, 1976). However, in all these studies judgments of stereoscopic depth could result from only partial dominance during binocular rivalry (Blake et al., 1991). So, it is possible that stereoscopic depth and rivalry are occurring at different spatial locations, giving the impression of coexistence.

Our aim was to determine whether a monocular stimulus whose appearance was reported as being completely suppressed from awareness could contribute to the perception of stereoscopic depth. Our stimulus involved judging the relative depth of grating patches that were presented at different binocular disparities. The grating patches in the two eyes differed in spatial frequency, orientation, spatial phase, and motion, so that they engaged in binocular rivalry on the majority of trials. Participants were asked to make stereoscopic depth judgments and then immediately report the perceptual appearance of the stimuli. Only trials in which the form from one eye dominated perception were used to generate stereoacuity thresholds.

\section{MATERIALS AND METHODS STIMULI}

Stimuli were programmed using a VSG2/5 graphics card (CRS, Rochester, England) and presented on a monochrome monitor with a fast phosphor decay (Clinton Monoray) and a frame-rate of $120 \mathrm{~Hz}$. Gamma correction was used to ensure that the monitor 
was linear over the entire luminance range used in the experiments. Participants viewed the display in a darkened room at a distance of $2.28 \mathrm{~m}$ through ferro-electric shutter-goggles (CRS, Rochester, England), which alternately occluded the two eyes at the same frequency as the frame-rate of the monitor. Thus, successive frames were seen by only one eye with no perceptible flicker at this high alternation rate. Participants fixated on a dark spot that remained visible throughout the experiments. In all of the experiments reported here, stimuli were circular patches of sinusoidally modulated grating (contrast, $40 \%$ ) with a $0.8^{\circ}$ hard edge envelope on a background of average luminance. Responses were recorded via a CB3 response box (CRS, Rochester, England). Participants were experienced psychophysical observers and had normal or corrected-to-normal vision and good stereopsis.

\section{PSYCHOMETRIC PROCEDURE}

Stereoacuity thresholds were first determined for monocular stimuli that differed in spatial frequency. To increase the number of trials in which full dominance was reported, we used a technique known as flash suppression (Wolfe, 1984; Holmes et al., 2006; see http://www.scholarpedia.org/article/Flash_suppression for a demonstration). First, an identical adapting stimulus was presented to both eyes. The adapting stimulus was a vertical grating patch in the center of the display with a spatial frequency between 1.5 and 6.0 cycles/deg. Participants adapted to the form/appearance of this grating patch for $1 \mathrm{~s}$. This was followed by a 1.5-s binocular presentation of three vertically arranged patches of grating. Figure 1 shows the spatial layout of the stimuli. On each trial, the spatial frequency of the gratings in one eye was 3 cycles/deg, while the spatial frequency of the gratings in the other eye varied between 1.5 and 6.0 cycles/deg. The spatial frequency of the gratings presented to one eye was identical to the adaptor. The top and bottom of the three grating patches in each monocular image were given opposite horizontal disparities such that one or other was in front of fixation during the stereo presentation. The central patch was always at zero disparity. Horizontal jitter \pm 5 arc min was applied independently to the spatial position of the top and bottom patches. This prevented the use of monocular cues to determine depth. The task of the observers was to press a button to indicate which grating patch (top or bottom) was closest. Immediately after their stereo judgment, observers were asked to indicate which grating patches were perceptually dominant. Trials in which full dominance was not reported for all grating patches in one eye and for the duration of the presentation were discarded from further analysis. Participants were easily able to detect differences between 3 cycles/deg patch and all spatial frequencies that were used.

Next, observers judged stereoscopic depth for monocular stimuli that differed in orientation, spatial phase, and direction of motion. In orientation blocks, participants viewed an identical adapting grating patch in the center of the screen that was either vertical, or tilted to the left or right of vertical $\left(5^{\circ}\right.$ or $\left.7.5^{\circ}\right)$. This was followed by a stereo presentation of three vertically aligned grating patches with the same stimulus parameters to one eye and gratings with an opposite tilt to the other. Participants determined the relative depth of the grating patches and then reported whether the patch was tilted to the right or left. In the spatial phase blocks,

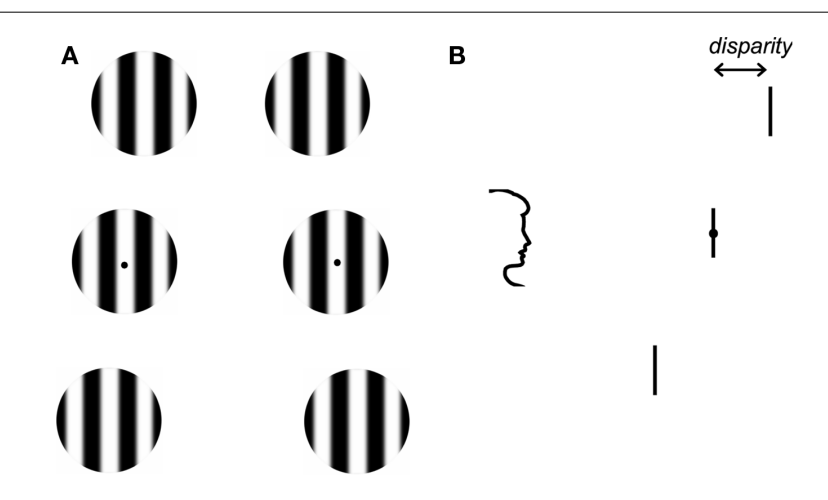

FIGURE 1 | Front and side views of the stimulus. (A) Stimuli were three vertically arranged grating patches. Each patch was $0.8^{\circ}$ in diameter and they were separated by $1^{\circ}$. The gratings presented to each eye could vary in either: spatial frequency, orientation, spatial phase, or direction of motion. (B) The top and bottom of the three grating patches in each monocular image were given equal and opposite horizontal disparities such that one or other was in front of fixation during the stereo presentation. The task of the participant was to press a button to indicate which grating patch was closest and then to report the appearance of the gratings.

the adapting stimulus was composed of a patch of vertical grating in the center of the display (spatial freq: 3 cycles/deg) with a spatial (cosine) phase of $0^{\circ}$. This was followed by a stereo presentation of three grating patches with the same spatial phase $\left(0^{\circ}\right)$ as the adaptor in one eye and grating patches with a spatial phase of either $0^{\circ}$ or $180^{\circ}$ to the other eye. Participants indicated depth and then indicated whether all grating patches had a spatial phase of $0^{\circ}$ (bright in the center) or $180^{\circ}$ (dark in the center). Finally, stereoacuity thresholds were determined for stimuli that differed in their direction of motion. In this experiment, observers viewed an identical adapting stimulus in the center of the display that was presented to both eyes for $1 \mathrm{~s}$. The adapting stimulus was composed of a patch of vertical gratings (spatial freq: 3 cycles/deg) with a temporal frequency of $0.5 \mathrm{cycle} / \mathrm{s}$ to the left or to the right. This was followed by a 1.5 -s stereo presentation of three grating patches with the same direction of motion as the adaptor in both eyes or with opposite directions-of-motion in each eye. Participants indicated depth and then indicated the perceived direction of movement of the gratings in the patches.

Stereoacuity functions in each experiment were based on five repetitions of a test block for each observer. Each test block contained 10 disparity steps for each stimulus combination and each stereo judgment was repeated five times. Within each test block, stimulus combinations were varied in a counterbalanced design, so that each stimulus was presented an equal number of times to the right and left eyes. For threshold discrimination, cumulativeGaussian curves were fitted to the data. The difference between performance at 0.25 and 0.75 was taken as the threshold.

\section{RESULTS}

\section{SPATIAL FREOUENCY}

Observers reported the complete dominance of all grating patches in one eye for the duration of the stimulus in over $90 \%$ of trials (S1: $96.1+3.1 \%$; S2: $93.7+3.1 \%$; S3: $95+1.7 \%)$. Trials in which the gratings from one eye or the other did not dominate 


\section{(1) (I)}

\section{(1) (II)}

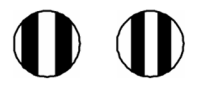

(II) (I)

(II) (I)
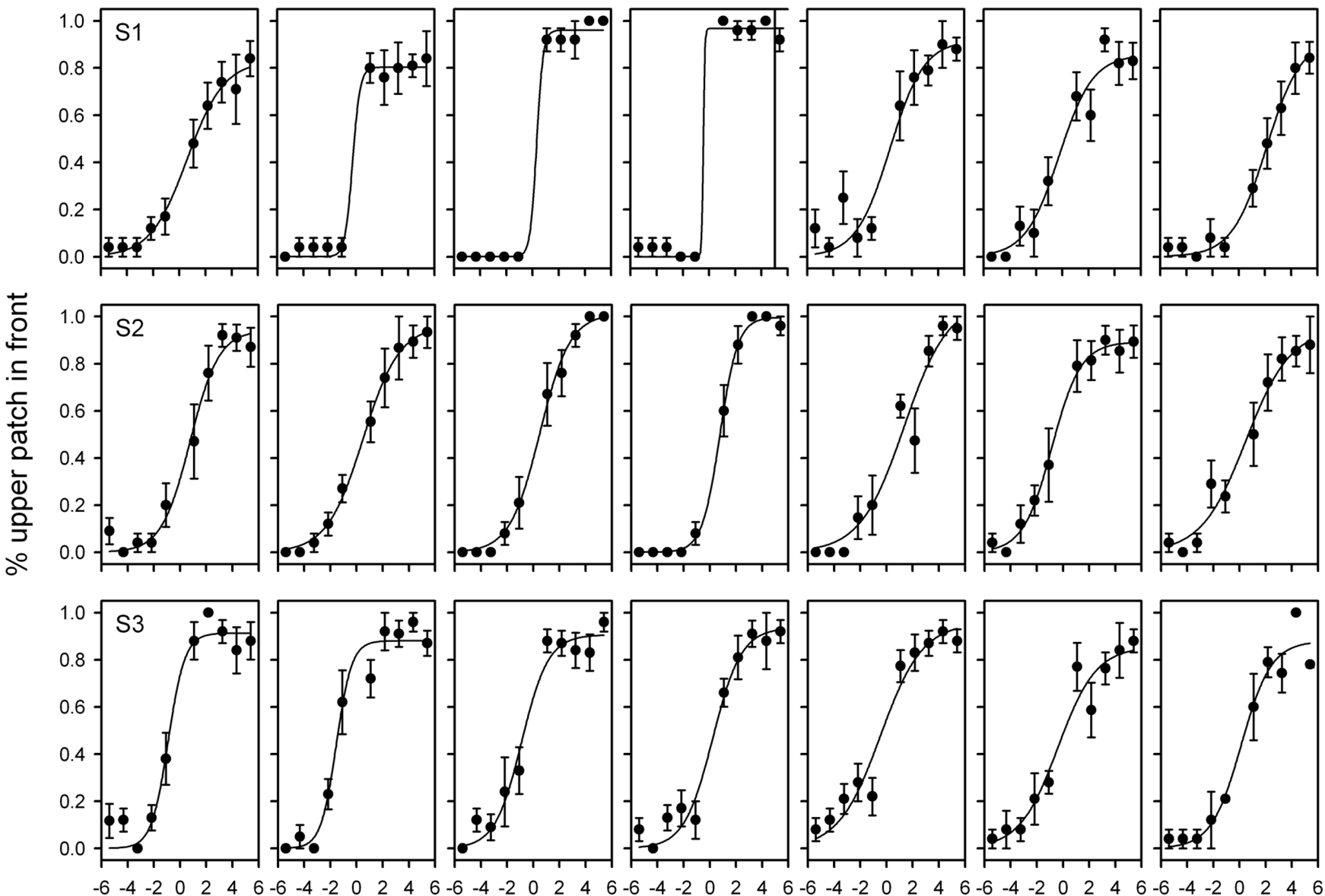

Disparity of lower patch (arc min)
FIGURE 2 | Stereoacuity functions for grating stimuli patches with different spatial frequencies in the two eyes. The combination of spatial frequencies used from (left to right) were: 1.5:3.0, 1.9:3.0, 2.4:3.0; 3.0:3.0;
3.8:3.0; 4.9:3.0; 6.0:3.0 cycles/deg. Participants were asked to indicate whether the upper or lower grating patch was closest. A negative value on the $x$ axis represents an uncrossed disparity. Bars represent SEM. exclusively were not analyzed. Therefore, with the exception of when both gratings had the same spatial frequency, stereoscopic judgments were made when the form from only one eye was visible. Figure 2 shows the stereoscopic depth functions from three observers. Thresholds were lowest when the gratings had the same spatial frequency (3.0: 3.0 cycles/deg: mean + SEM $=1.47+0.59)$ and increased as the spatial frequency of the gratings were made more different from each other $(1.5: 3.0=2.83+0.64 ; 1.9$ : $3.0=1.93+0.54 ; 2.4: 3.0=1.90+0.53 ; 3.8: 3.0=3.13+0.28 ; 4.9$ : $3.0=3.00+0.31 ; 6.0: 3.0=3.43+0.23)$. An ANOVA showed that there was an effect of spatial frequency for $S 1[F(1,6)=4.3$, $p<0.005]$, but not for S2 $(F=0.71, p=0.64)$ or S3 $(F=0.92$, $p=0.49$ ). Nevertheless, the key point is that clear psychometric functions are apparent for each observer when the spatial frequency of the two monocular images differed by as much as a factor of two.

\section{ORIENTATION}

Next, stereoacuity thresholds were determined for grating patches that varied in orientation from $0^{\circ}$ to $15^{\circ}$ (Figure 3A). When the orientations of the two gratings were different, participants indicated complete suppression of the form of the gratings presented to one eye or the other in about $90 \%$ of trials (S1: $88.6+1.6 \%$; S2: $89.0+2.2 \%)$. Stereoscopic depth judgments were only assessed in these trials. The results, shown in Figure 3, show that thresholds were lowest when the grating patches had the same orientation (i.e., vertical) and increased as they were made more different from each other. An ANOVA revealed that there was a significant effect of orientation for S1 $(F=5.9, p<0.05)$, but not for S2 $(F=2.8, p=0.1)$. However, again both observers were still able to generate good stereoscopic depth functions when the content of the grating patches in one eye was suppressed from perception. 


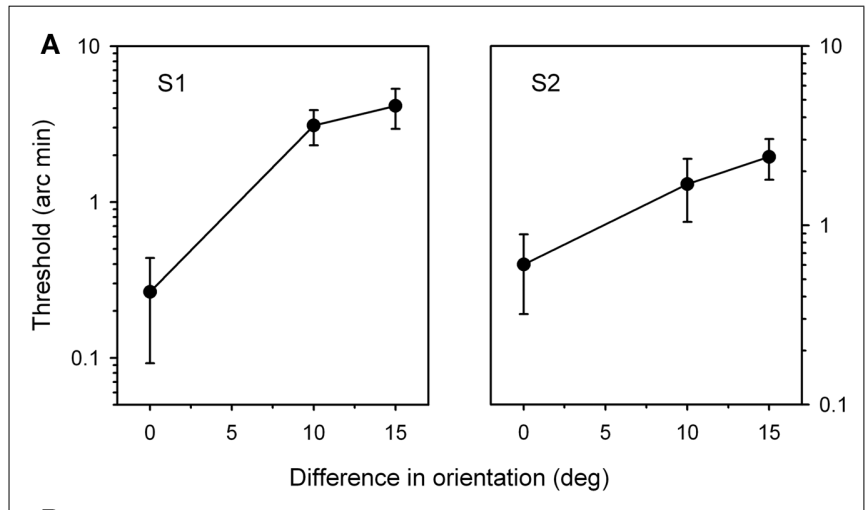

B
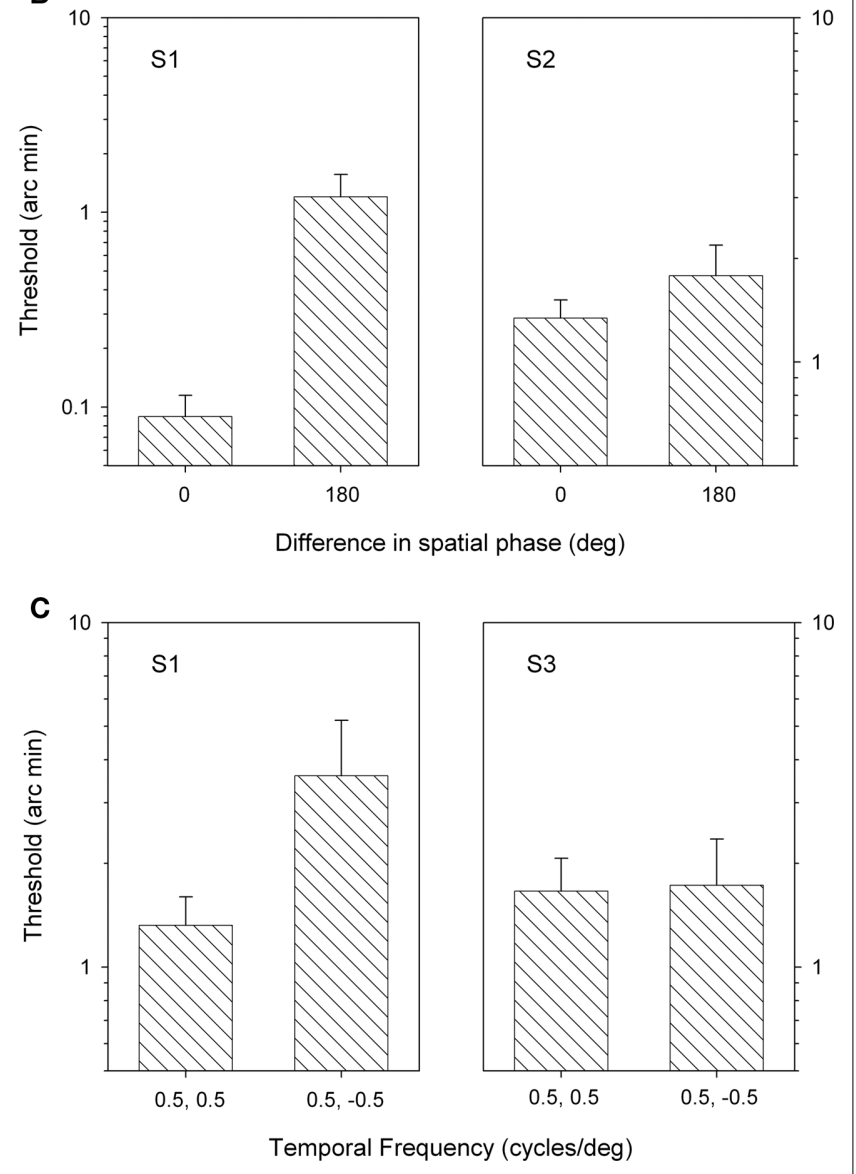

FIGURE 3 | Stereoacuity thresholds for grating patches that varied in orientation (A), spatial phase (B), or direction of motion (C). Bars represent SEM.

\section{SPATIAL PHASE}

Stereoscopic depth judgments were then made when grating stimuli differed in their spatial phase. Clear dominance of one or other stimulus was apparent in over $90 \%$ of the $180^{\circ}$ phase different trials (S1: $92.0+5.0 \%$; S2: $97.2+1.2 \%$ ). Figure 3B shows that thresholds were lower when both sets of gratings had the same spatial phase $\left(0^{\circ}\right)$ compared to when the gratings were $180^{\circ}$ out of phase $\left(0^{\circ}\right.$ and $\left.180^{\circ}\right)$. This difference was significant for S1 $(t=9.4$, $p<0.05)$, but not for S2 $(t=1.9, p=0.20)$. Nonetheless, reliable stereoscopic depth functions were still obtained when the form of one image was suppressed from awareness.

\section{DIRECTION OF MOTION}

Finally, stereoacuity thresholds were determined for stimuli that differed in their direction of motion. Participants reported complete dominance of one spatial frequency or the other across all three grating patches for the duration of the presentation on over $40 \%$ of trials (S1: $97 \pm 0.9 \%$; S3: $48 \pm 6.6 \%$ ). Figure $3 \mathrm{C}$ shows the stereoacuity thresholds from two participants. For one participant, thresholds were significantly lower when the gratings had the same direction of motion and increased when the direction of motion of the gratings was in the opposite directions. However, this difference was not statistically significant $\mathrm{S} 1(t=2.5$, $p=0.16)$. There was not difference in the stereo-thresholds for S3 $(t=0.01, p=0.93)$. Again, stereo judgments were still possible even when the direction of motion of one stimulus was completely suppressed from perception.

\section{DISCUSSION}

The aim of this study was to determine whether depth judgments based on binocular disparity can occur for two monocular objects that differ in spatial structure or local motion. Our results show that stereopsis is still possible when the appearance of an object from one eye is completely suppressed from awareness during binocular rivalry.

A number of previous studies have reported that stereoscopic depth perception can coexist with binocular rivalry (Treisman, 1962; Ogle and Wakefield, 1967; Julesz and Miller, 1975; Harrad et al., 1994). However, in all of these studies participants were not asked to report whether the image in the "suppressed" eye was perceptually dominant during judgments of depth. So, it is possible that, in these studies, partial dominance could result in stereoscopic depth and rivalry occurring at different spatial locations, giving the impression of coexistence. These problems were circumvented in the present study by having participants report the depth and appearance of the stimulus on each trial. Only those trials in which the form from one eye dominated perception for the duration of the trial were used to generate stereoacuity thresholds.

The coexistence of stereopsis and binocular rivalry in this study demonstrates that binocular disparity information can be processed even when the appearance of one monocular image has been suppressed from awareness. These findings fit with a recent study that showed binocular integration and suppression are possible when vertical gratings were presented to each eye (Su et al., 2009). In this study, the stimuli in the two eyes were identical gratings except that a circular patch in the center of the vertical grating of one eye was phase-shifted relative to the surrounding grating. This generated both local rivalry and disparity signals. Although participants used the disparity information to generate a perception of stereoscopic depth, thresholds for the detection of a probe stimulus at the location of the circular patch in corresponding regions of the other eye were increased. This clearly demonstrates the coexistence of binocular integration and suppression. Interestingly, the stimulus used by Su et al. (2009) is similar to the phase-shifted stimulus used in this experiment 
(Figure 3B). However, in this experiment, we used the participant's reports of awareness to indicate interocular suppression.

The illusory conjunction of form and depth reported in this study fits with other reports that have shown that independent competition between different features of a stimulus can occur during binocular rivalry. For example, a suppressed stimulus can influence the appearance of the dominant stimulus by changing the perception of its orientation (Pearson and Clifford, 2005), direction of motion (Andrews and Blakemore, 2002), luminance (Carlson and He, 2000), and color (Carney et al., 1987; Holmes et al., 2006; Hong and Shevell, 2009). The implication from these studies is that the neural mechanisms underlying suppression during binocular rivalry can operate independently on the features that make up the stimulus. These findings suggest that a stimulus that is suppressed during binocular rivalry is not equivalent to physical removal of the stimulus. Rather, the stimulus can continue to influence perception. These findings are consistent with the idea that the suppression of information during rivalry is not an all or nothing process, but one that occurs at multiple sites throughout the visual system (Blake and Logothetis, 2002; Tong et al., 2006; Blake and Wilson, 2011).

Circumstances in which rivalry and stereopsis coexist are common in natural viewing. For example, when we view a $3 \mathrm{D}$ scene, occluding objects typically generate images in corresponding regions of the two eyes that are different (Anderson and Nakayama, 1994). However, if these rivalrous zones are consistent with viewing an occluding object, they are perceived at an appropriate depth (Shimojo and Nakayama, 1990). In this study, when a disparity was applied to the images, the rival stimuli in the two eyes did not occupy corresponding retinal points throughout. Nevertheless, when one grating patch dominated perception, it always appeared as a single circular patch. It would appear, therefore, that it is possible for binocular rivalry to take place even when some aspects of the rival stimuli occupy non-corresponding regions of visual space. Presumably, information from stereoscopic processing can influence which regions of the retina interact during rivalry.

\section{REFERENCES}

Alais, D., and Blake, R. (2005). Binocular Rivalry. Cambridge, MA: MIT Press.

Anderson, B. L., and Nakayama, K. (1994). Towards a general theory of stereopsis: binocular matching, occluding contours and fusion. Psychol. Rev. 101, 413-445.

Andrews, T. J., and Blakemore, C. (2002). Integration of motion information during binocular rivalry. Vision Res. 42, 301-309.

Anzai, A., Ohzawa, I., and Freeman, R. D. (1997). Neural mechanisms underlying binocular fusion and stereopsis: position vs phase. Proc. Natl. Acad. Sci. U.S.A. 94, 5438-5443.

Blake, R. (1989). A neural theory of binocular rivalry. Psychol. Rev. 96, 145-167.
Blake, R., and Logothetis, N. K. (2002). Visual competition. Nat. Rev. Neurosci. 3, 1-11.

Blake, R., and Wilson, H. (2011). Binocular vision. Vision Res. 51, 754-770.

Blake, R., Yang, Y., and Wilson, H. R. (1991). On the coexistence of stereopsis and binocular rivalry. Vision Res. 31, 1191-1203.

Carlson, T. A., and He, S. (2000). Visible binocular beats from invisible stimuli during rivalry. Curr. Biol. 10, 1055-1058.

Carney, T., Shadlen, M., and Switkes, E. (1987). Parallel processing of motion and colour information. Nature 328, 647-649.

Cogan, A., Kontsevich, L. L., Lomakin, A. J., Halpern, D. L., and Blake, R. (1995). Binocular disparity processing with opposite-contrast stimuli. Perception 24, 33-47.
Although our results show that stereo-depth is possible when the monocular input differs on a variety of stimulus dimensions. A number of other reports have shown that reducing the correspondence in spatial structure between the two monocular images affects stereopsis. For example, adding masking noise at one spatial frequency impairs stereoscopic depth at similar, but not at different spatial frequencies (Julesz and Miller, 1975; Mayhew and Frisby, 1976; Yang and Blake, 1991). Neurophysiological studies have shown that there are two possible mechanisms that the visual system could use to process binocular disparity information (Cumming, 1997; Blake and Wilson, 2011). One model is based on binocular detectors, with identical receptive field structures, located at different locations for the left and right eyes (Anzai et al., 1997). An opposing model proposes that the envelope enclosing the receptive fields in the right and left eye are in corresponding retinal positions, but have a different spatial structure (DeAngelis et al., 1991). These mechanisms are based on first-order mechanisms that involve detecting corresponding luminance profiles in the two eyes. In our paradigm, the first-order or luminance pattern of the rivalrous stereograms did not match in the two eyes. This suggests that a second-order mechanism is involved. Behavioral evidence for second-order stereo-depth mechanism has been shown in a number of studies (Zeigler and Hess, 1999; Hess and Wilcox, 2008). Stereoscopic depth perception can even be obtained with dichoptically mixed first- and second-order stimuli (Edwards et al., 2000). Physiological support for second-order depth perception is evident in a subset of neurons in extrastriate regions of cat visual cortex (Tanaka and Ohzawa, 2006). Our findings suggest that the second-order stereo mechanisms can still operate during binocular rivalry.

In conclusion, our results show that a stimulus that is completely suppressed from awareness during binocular rivalry can nonetheless contribute to the processing of disparity. This gives rise to an illusory conjunction in which form information from one eye is combined with depth information from both eyes. These results demonstrate a range of stimulus conditions in which rivalry and stereopsis can coexist.

Cumming, B. G. (1997). Stereopsis: how the brain sees depth. Curr. Biol. 7, R645-R647.

DeAngelis, G. C., Ohzawa, I., and Freeman, R. D. (1991). Depth is encoded in the visual cortex by a specialized receptive field structure. Nature 352 , 156-159.

Edwards, M., Pope, D. R., and Schor, C. M. (2000). First- and second-order processing in transient stereopsis. Vision Res. 40, 2645-2651.

Harrad, R. A., McKee, S. P., Blake, R., and Yang, Y. (1994). Binocular rivalry disrupts stereopsis. Perception 23, 15-28.

Hess, R. F., and Wilcox, L. M. (2008). The transient nature of 2nd-order stereopsis. Vision Res. 48, 1327-1334.

Holmes, D., Hancock, S., and Andrews, T. J. (2006). Independent binocular integration for form and colour. Vision Res. 46, 665-677.
Hong, S. W., and Shevell, S. K. (2009). Color-binding errors during rivalrous suppression of form. Psychol. Sci. 20, 1084-1091.

Julesz, B. (1971). Foundations of Cyclopean Perception. Chicago: University of Chicago Press.

Julesz, B., and Miller, J. E. (1975). Independent spatial-frequency-tuned channels in binocular fusion and rivalry. Perception 4, 125-143.

Lehky, S. R., and Blake, R. (1991). Organization of binocular pathways: modeling and data related to rivalry. Neural Comput. 3, 44-53.

Mayhew, J. E. W., and Frisby, J. P. (1976). Rivalrous texture stereograms. Nature 264, 53-56.

Ogle, K. N., and Wakefield, J. M. (1967). Stereoscopic depth and binocular rivalry. Vision Res. 7, 89-98.

Parker, A. (2007). Binocular depth perception and the cerebral 
cortex. Nat. Rev. Neurosci. 8, Tanaka, H., and Ohzawa, I. (2006). 379-391.

Pearson, J., and Clifford, C. W. G. (2005). When your brain decides what you see: grouping across monocular, binocular and stimulus rivalry. Psychol. Sci. 16, 516-519.

Shimojo, S., and Nakayama, K. (1990). Real world occlusion constraints and binocular rivalry. Vision Res. 35, 179-195.

Su, Y., He, Z. J., and Ooi, T. L. (2009). Coexistence of binocular integration and suppression determined by surface border information. Proc. Natl. Acad. Sci. U.S.A. 106, 15990-15995. Neural basis for stereopsis from second-order contrast cues. J. Neurosci. 26, 4370-4382.

Tong, F., Meng, M., and Blake, R. (2006). Neural bases of binocular rivalry. Trends Cogn. Sci. 10, 502-511.

Treisman, A. (1962). Binocular rivalry and stereoscopic depth perception. Q. J. Exp. Psychol. 14, 23-29.

Wheatstone, C. (1838). Contributions to the physiology of vision - part the first: on some remarkable, and hitherto unobserved, phenomena of binocular vision. Philos. Trans. $R$. Soc. Lond. B Biol. Sci. 128, 371-394.

Wolfe, J. M. (1984). Reversing ocular dominance and suppression in a single flash. Vision Res. 24, 471-478.
Wolfe, J. M. (1986). Stereopsis and binocular rivalry. Psychol. Rev. 93 , 269-282.

Yang, Y., and Blake, R. (1991). Spatial frequency tuning of human stereopsis. Vision Res. 31, 1177-1189.

Zeigler, L. R., and Hess, R. F. (1999). Stereoscopic depth but not shape perception from second-order stimuli. Vision Res. 39, 1491-1507.

Conflict of Interest Statement: The authors declare that the research was conducted in the absence of any commercial or financial relationships that could be construed as a potential conflict of interest.
Received: 01 July 2011; paper pending published: 18 July 2011; accepted: 23 August 2011; published online: 21 September 2011.

Citation: Andrews TJ and Holmes $D$ (2011) Stereoscopic depth perception during binocular rivalry. Front. Hum. Neurosci. 5:99. doi: 10.3389/fnhum.2011.00099

Copyright (C) 2011 Andrews and Holmes. This is an open-access article subject to a non-exclusive license between the authors and Frontiers Media SA, which permits use, distribution and reproduction in other forums, provided the original authors and source are credited and other Frontiers conditions are complied with. 